\title{
Test yourself answer to question: a 77-year-old female presented with a 3-week history of left thigh swelling and pain
}

\author{
Susan Hesni ${ }^{1} \cdot$ Davoud Khodatars $^{1} \cdot$ Ramanan Rajakulasingam $^{1}{ }^{10} \cdot$ Asif Saifuddin $^{1} \cdot$ Paul O'Donnell $^{1}$
}

Received: 1 September 2021 / Revised: 9 November 2021 / Accepted: 11 November 2021 / Published online: 27 January 2022

(c) Crown 2022

\begin{abstract}
Answer:
Proliferative myositis of the left adductor magnus muscle Discussion:

MRI (Fig. 1a) demonstrates extensive muscle oedemalike signal within the medial aspect of adductor magnus. Poorly defined low SI, limited to the muscle belly with surrounding oedema-like signal is also seen but the muscle fibres are not replaced or disrupted (Fig. 1b). Subsequent US (Fig. 2a) shows prominent hypoechoic bands between swollen hyperechoic muscle fascicles - the 'dry cracked mud' appearance described in proliferative myositis (PM). Longitudinal images showed continuous, hyperechoic muscle fibres without a focal mass. US guided biopsy was performed with histology revealing skeletal muscle with focal degeneration and spindle cells. Repeat US only 2 -weeks later confirmed the mass had already reduced in size, muscle fibres remained continuous and that there was subtle return of normal muscle fibre echotexture (Fig. 2b). MRI at 3 months shows complete lesion resolution, by which time symptoms had also resolved.

$\mathrm{PM}$ is a benign pseudo-sarcomatous process within the spectrum of fibroblastic/myofibroblastic tumours. It is usually seen in middle aged adults (median age 50 years [1]),
\end{abstract}

The case presentation can be found at https://doi.org/10.1007/ s00256-021-03959-6

Ramanan Rajakulasingam

mramanan.rajakulasingam1@nhs.net

Susan Hesni

Susan.hesni@nhs.net

Davoud Khodatars

davoud.khodatars1@nhs.net

Asif Saifuddin

asif.saifuddin@nhs.net

Paul O'Donnell

paulodonnell@nhs.net

1 Department of Radiology, Royal National Orthopaedic Hospital, Brockley Hill, Stanmore HA7 4LP, UK but cases in the paediatric population have been described [1-3]. The typical presentation is of a painful, rapidly growing soft tissue mass, most often located in the upper limbs [1]. Its aetiology is not clear but an association with trauma, inflammatory and ischaemic processes have been postulated [1-3].

PM is characterized by the presence of ganglion-like cells and proliferative spindle cells interposed between preserved muscle fibres which can give a 'checkerboard' pattern histologically $[2,3]$.

MRI typically shows mass-like swelling of the muscle, which is generally hyperintense, often homogeneous, on T2 / fluid sensitive sequences and hypo- to isointense on $\mathrm{T} 1$, with perilesional oedema-like signal and homogenous enhancement, commonly extending to the surrounding fascia [4]. Intermediate to low T2 SI linear foci, analogous to the checkerboard pattern seen histologically, and occasionally larger areas of T2 hypointensity [3] may be seen. Muscle fibres remain continuous throughout the lesion, differentiating it from a soft tissue sarcoma (STS). The inflammatory appearance and frequent linear hypointensity is also atypical for STS. US confirms muscle continuity and may demonstrate fascial infiltration with occasional increased vascularity on colour Doppler. The 'cracked dry mud' sign describes the US appearance of hypoechoic bands between swollen hyperechoic muscle fibres: this is a highly specific sign for PM [5] but has also rarely been described in early myositis ossificans [6].

PM is a self-limiting process with spontaneous regression (usually within 1 year), without tendency to recur [7]. Conservative treatment is preferred. In addition to sarcoma, the main differential diagnosis is nodular fasciitis which appears as a solid mass on imaging, although cystic and myxoid components have been described. Myositis ossificans typically exhibits a low signal curvilinear lesion with intense surrounding oedema in the early stages [6]. In nodular intramuscular sarcoid, lesions may have a star shaped low central surrounded by high SI, so called 'dark star sign' [8]. 
Fig. 1 Axial PD SPAIR MR image of the left thigh (a) shows an ill-defined lesion within the left adductor magnus muscle, consisting of linear / reticular high signal (arrows) surrounding a central area of hypointensity (*). b Sagittal T2W FSE image shows elongated muscle hyperintesity with central low signal (*), both images showing intact muscle fibres
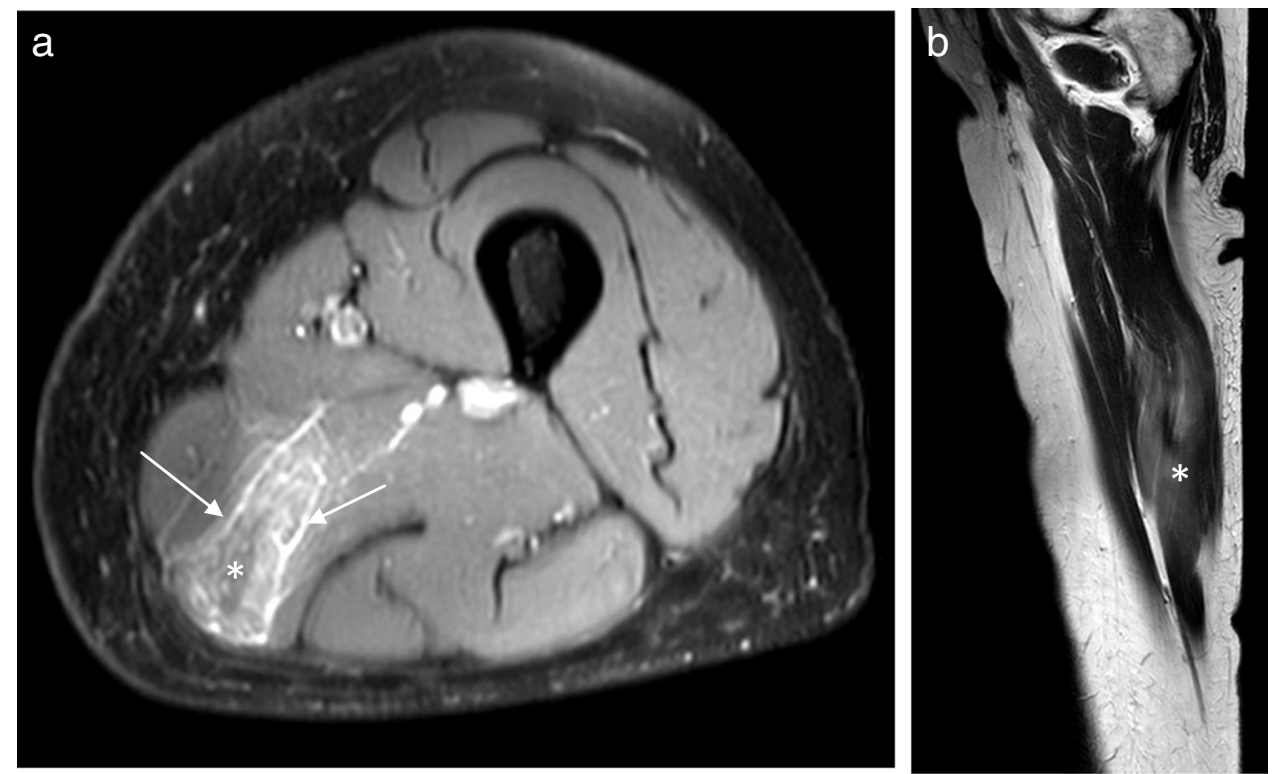
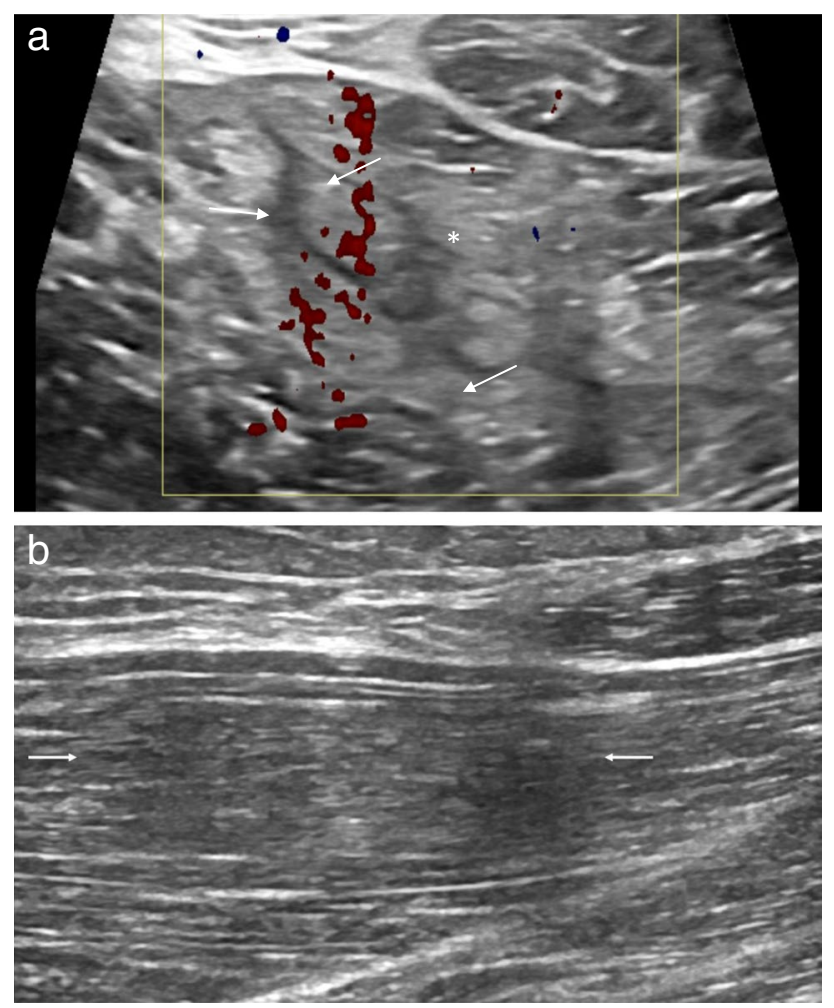

Fig. 2 Transverse US image of the left thigh during initial biopsy (a) shows multiple hypoechoic bands (arrows) separating swollen, hyperechoic adductor magnus muscle fibres $(*)$, giving a 'dry cracked mud,' appearance. (The Doppler signals shown are artefactual and no internal neovascularity was present). Longitudinal US image on a follow up study only 2-weeks later (b) shows reduced muscle bundle swelling, subtle return of the normal echotexture and confirms continuity of muscle fibres (arrows)
An astute radiologist may suggest the diagnosis of PM if the US and MRI findings mentioned above are recognized. A sarcoma should be excluded on biopsy, and it should be possible to avoid surgery if clinical / radiological surveillance confirms resolution.

\section{Declarations}

Conflict of interest The authors declare that they have no conflicts of interest.

\section{References}

1. Enzinger FM, Dulcey F. Proliferative myositis. Report of thirtythree cases. Cancer. 1967;20(12):2213-23.

2. Gan S, Xie D, Dai H, Zhang Z, Di X, Li R, Guo L, Sun Y. Proliferative myositis and nodular fasciitis: a retrospective study with clinicopathologic and radiologic correlation. Int J Clin Exp Pathol. 2019;12(12):4319-28.

3. Talbert RJ, Laor T, Yin H. Proliferative myositis: expanding the differential diagnosis of a soft tissue mass in infancy. Skelet Radiol. 2011;40(12):1623-7.

4. Wlachovska B, Abraham B, Deux JF, Sibony M, Marsault C, Le Breton C. Proliferative myositis in a patient with AIDS. Skelet Radiol. 2004;33(4):237-40.

5. Pagonidis K, Raissaki M, Gourtsoyiannis N. Proliferative myositis: value of imaging. J Comput Assist Tomogr. 2005;29(1):108-11.

6. Wang H, Nie P, Li Y, Hou F, Dong C, Huang Y, Hao D. MRI findings of early myositis Ossificans without calcification or ossification. Biomed Res Int. 2018;2018:4186324.

7. Kern WH. Proliferative myositis; a pseudosarcomatous reaction to injury: a report of seven cases. Arch Pathol. 1960;69:209-16. 
8. Ganeshan D, Menias CO, Lubner MG, Pickhardt PJ, Sandrasegaran K, Bhalla S. Sarcoidosis from head to toe: what the radiologist needs to know. Radiographics. 2018;38(4):1180-200.
Publisher's note Springer Nature remains neutral with regard to jurisdictional claims in published maps and institutional affiliations. 